\title{
Flat bending shape stability of the beams with variable section width
}

\author{
Anastasia Lapina ${ }^{1}$, Serdar Yazyev $^{1}$, Anton Chepurnenko ${ }^{1,}$, , and Irina Dubovitskaya ${ }^{2}$ \\ ${ }^{1}$ Don State Technical University, 1 Gagarin sq., Rostov-on-Don, 344000, Russia \\ ${ }^{2}$ Russian State Tourism and Service University, 99 Glavnaya street, Moscow, 141221, Russia
}

\begin{abstract}
The paper proposes a methodology for calculating lateral buckling of beams of variable rectangular cross section based on the energy approach. The technique is considered on the example of a cantilever beam of variable width with two sections under the action of a concentrated force. The twist angle function was set in the form of a trigonometric series. As a result, the problem is reduced to a generalized secular equation.
\end{abstract}

\section{Introduction}

When designing beams with a narrow rectangular cross-section, it is necessary to check them for stability of a flat bending shape. In construction, beam structures with a constant geometry are widely used, and to reduce the consumption of material it is advisable to use beams of variable stiffness. The calculation of beams of variable cross section compared with the calculation of structures of constant stiffness is associated with more mathematical difficulties. In $[1,2]$, solutions are given for beams whose geometric characteristics are continuous functions of coordinates. If the functions of changing the rigidity of the structure have discontinuities, it becomes necessary to satisfy the boundary conditions at each joint of the sections. In this case, it is convenient not to directly solve the differential equilibrium equations, but to use variational methods [3].

\section{Methods}

We will consider the calculation method on the example of a beam with two sections (Figure 1). The following notation is introduced:

$$
\frac{b_{2}}{b_{1}}=\gamma ; \quad \frac{b_{1}}{h}=\delta
$$

\footnotetext{
* Corresponding author: anton chepurnenk@mail.ru
} 


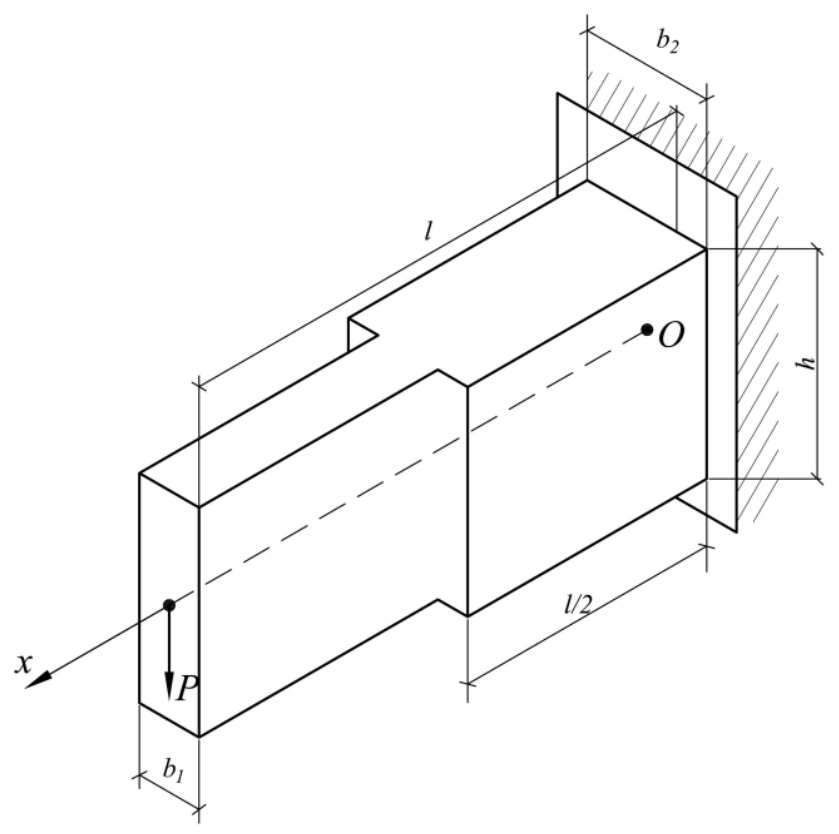

Fig. 1. The beam under consideration

The ratio of the stiffnesses of the sections to bend in the plane of least stiffness is calculated by the formula:

$$
\frac{E I_{z 2}}{E I_{z 1}}=\frac{b_{2}^{3} h / 12}{b_{1}^{3} h / 12}=\gamma^{3} .
$$

The ratio of torsional stiffnesses of the sections is calculated as:

$$
\frac{G I_{t 2}}{G I_{t 1}}=\frac{\frac{h b_{2}^{3}}{3}\left(1-0.63 \frac{b_{2}}{h}\right)}{\frac{h b_{1}^{3}}{3}\left(1-0.63 \frac{b_{1}}{h}\right)}=\gamma^{3} \frac{1-0.63 \delta \gamma}{1-0.63 \delta}=\alpha .
$$

To solve, we use the energy method in the formulation presented in [4].The critical load is determined from the condition of minimum total energy $U$ representing the difference between the potential strain energy $W$ and the work of external forces $A$ :

$$
U=W-A \text {. }
$$

The potential strain energy is the sum of the potential energy of bending and torsion and, in the case of variable stiffness, is determined by the formula:

$$
W=\frac{1}{2} \int_{0}^{l} E I_{z}(x)\left(\frac{d^{2} v}{d x^{2}}\right)^{2} d x+\frac{1}{2} \int_{0}^{l} G I_{t}(x)\left(\frac{d \theta}{d x}\right)^{2} d x
$$

where $v$ is the deflection and $\theta$ is the twist angle.

The work of external forces $A$ is defined as [5]: 


$$
A=-\int_{0}^{l} M_{y} \theta \frac{d^{2} v}{d x^{2}} d x
$$

where $M_{\mathrm{y}}$ is the bending moment for the beam under consideration, determined by the formula:

$$
M_{y}(x)=-P l+P x=P l(\xi-1) .
$$

In formula (7), $\xi=x / l$ represents the dimensionless coordinate.

At the moment of lateral buckling, the relationship between the second derivative of the deflection, the bending moment, and the twist angle has the form [6]:

$$
\frac{d^{2} v}{d x^{2}}=-\frac{M_{y} \theta}{E I_{z}}
$$

Substituting (8) in (6), and then (6) and (5) in (4), we obtain:

$$
U=\frac{1}{2} \int_{0}^{l} G I_{t}(x)\left(\frac{d \theta}{d x}\right)^{2} d x-\frac{1}{2} \int_{0}^{l} \frac{M_{y}^{2} \theta^{2}}{E I_{z}(x)} d x .
$$

For the beam shown in Figure 1, expression 9 takes the form:

$$
\begin{aligned}
& U=\frac{1}{2}\left(G I_{t 1} \int_{0}^{0.5 l}\left(\frac{d \theta}{d x}\right)^{2} d x+G I_{t 2} \int_{0.5 l}^{l}\left(\frac{d \theta}{d x}\right)^{2} d x-\right. \\
& \left.-\frac{1}{E I_{z 1}} \int_{0}^{0.5 l} P^{2}(l-x)^{2} \theta^{2} d x-\frac{1}{E I_{z 2}} \int_{0.5 l}^{l} P^{2}(l-x)^{2} \theta^{2} d x\right) .
\end{aligned}
$$

We introduce the dimensionless quantity $\lambda$ by the formula:

$$
\lambda=\frac{P^{2} l^{4}}{G I_{t 1} E I_{z 1}} .
$$

Minimizing the functional $U$ is equivalent to minimizing the functional $\Lambda=\frac{U l}{G I_{t 1}}$ which is written as:

$$
\Lambda=\frac{1}{2}\left(\alpha \int_{0}^{0.5}\left(\frac{d \theta}{d \xi}\right)^{2} d \xi+\int_{0.5}^{1}\left(\frac{d \theta}{d \xi}\right)^{2} d \xi-\lambda\left[\frac{1}{\gamma^{3}} \int_{0}^{0.5}(\xi-1)^{2} \theta^{2} d \xi+\int_{0.5}^{1}(\xi-1)^{2} \theta^{2} d \xi\right]\right)
$$

The function of the twist angle will be sought in the form:

$$
\theta=a_{1} \sin \frac{\pi \xi}{2}+a_{2} \sin \frac{3 \pi \xi}{2}+a_{3} \sin \frac{5 \pi \xi}{2}+\ldots=\sum_{i=1}^{n} a_{i} \sin \frac{\pi(2 i-1) \xi}{2}
$$

Substituting (13) into (12) and minimizing the functional $\Lambda$ with unknown coefficients $a_{\mathrm{i}}$, we obtain a system of linear algebraic equations:

$$
([A]-\lambda[B])\{X\}=0,
$$


where $\{X\}=\left\{\begin{array}{llll}a_{1} & a_{2} & \ldots & a_{n}\end{array}\right\}^{T}-$ vector of series coefficients.

The coefficients $A_{i j}$ and $B_{i j}$ of the matrices $[A]$ and $[B]$ are determined by the formulas:

$$
\begin{aligned}
A_{i j}= & \frac{\pi^{2}}{4}\left(\alpha \int_{0}^{0.5}(2 i-1)(2 j-1) \cos \frac{\pi(2 i-1) \xi}{2} \cos \frac{\pi(2 j-1) \xi}{2} d \xi+\right. \\
& \left.+\int_{0.5}^{1}(2 i-1)(2 j-1) \cos \frac{\pi(2 i-1) \xi}{2} \cos \frac{\pi(2 j-1) \xi}{2} d \xi\right) . \\
B_{i j}= & \frac{1}{\gamma^{3}} \int_{0}^{0.5} \sin \frac{\pi(2 i-1) \xi}{2} \sin \frac{\pi(2 i-1) \xi}{2}(\xi-1)^{2} d \xi+ \\
& +\int_{0.5}^{1} \sin \frac{\pi(2 i-1) \xi}{2} \sin \frac{\pi(2 i-1) \xi}{2}(\xi-1)^{2} d \xi .
\end{aligned}
$$

Integration in formulas (15) can be performed numerically or using symbolic computation packages. The critical load is determined from the condition that the determinant of the system (14) is equal to zero:

$$
|[A]-\lambda[B]|=0 .
$$

The critical load is determined from (11) by the formula:

$$
P_{c r}=K \frac{\sqrt{G I_{t 1} E I_{z 1}}}{l^{2}},
$$

where $K=\sqrt{\lambda_{\min }}, \lambda_{\min }-$ minimum eigenvalue.

\section{Results and Discussion}

The test problem was solved for $\gamma=1.1, \delta=0.1$. Figure 2 shows a graph of the coefficient $K$ for a different number of members of the series. Good convergence is observed. The results of the fourth and fifth approximations practically coincide. With one member of the series, the solution differs from the exact one by $4.6 \%$.

The value of the coefficient $K$ with $n=4$ is 5.0386. With such initial data, the considered problem was solved earlier in [7], and there the value 5.03 was obtained for the coefficient $K$. The solution proposed in [7] is based on the direct integration of differential equations in each section while satisfying the conditions at the boundaries. With a large number of sections, the solution by this method is significantly complicated. Our proposed method is devoid of this drawback and allows you to get a solution for any number of sections. 


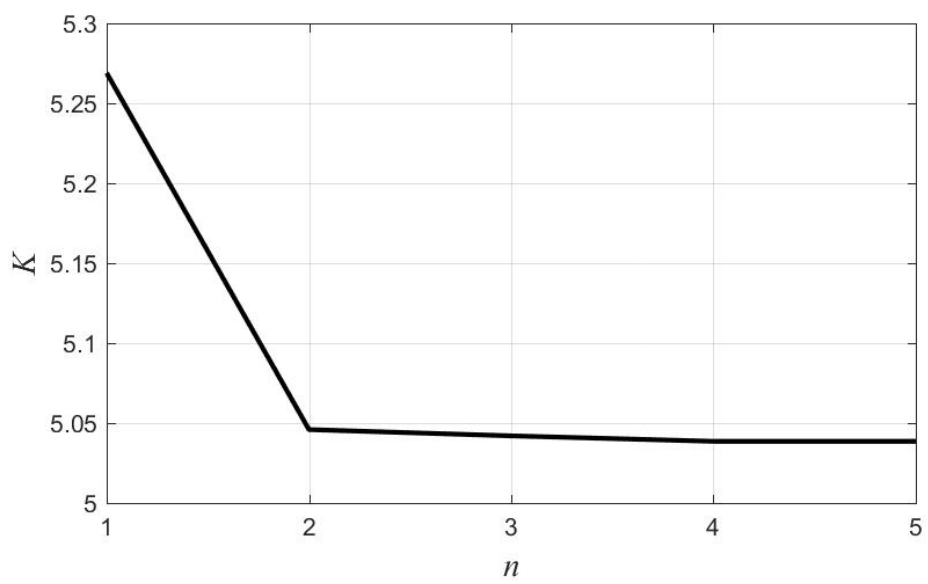

Fig. 2. The dependence of the coefficient $K$ on the number of members of the series

\section{Conclusions}

The resolving equation of the energy method for calculating lateral buckling of beams with a variable width of the rectangular cross section is obtained. The problem is reduced to a generalized secular equation. Good method convergence is demonstrated. The reliability of the results is confirmed by comparison with the solution of other authors. The proposed technique allows you to calculate the structures of variable stiffness for an arbitrary number of sections. In the considered problem, the vertical displacement of the load relative to the center of gravity is not taken into account. These issues for the beams with constant cross section are studied in more detail in the paper [8]. We also did not investigated the problem of the presence of initial imperfections and taking into account physical nonlinearity, which are considered in [9-11].

\section{References}

1. A.A. Karamisheva, S.B. Yazyev, A.A. Avakov, Procedia Engineering. 150, 1872-1877 (2016)

2. A. P. Lapina, I.M. Zotov, A.S. Chepurnenko, B.M. Yazyev, E3S Web of Conferences. 97, 04067 (2019)

3. S. Yazyev et al., E3S Web of Conferences. 97, 04066 (2019)

4. A. P. Lapina, I.M. Zotov, A.S. Chepurnenko, B.M. Yazyev, Vestnik Volgogradskogo gosudarstvennogo arkhitekturno-stroitel'nogo universiteta. Seriya: Stroitel'stvo i arkhitektura. 77, 5-16 (2019)

5. V.M. Makushin, N.G. Savel'yev, N. V. Khung. in: Raschety na prochnost. 14, 268-289 (1969)

6. S. P. Timoshenko, J. M. Gere, Theory of elastic stability (McGraw-Hill, 1961)

7. A.F. Smirnov et al., Structural mechanics. Dynamics and stability of structures (Stroizdat, 1984)

8. A. S. Chepurnenko, V.V. Ulianskaya, S.B. Yazyev, I.M. Zotov, MATEC Web of Conferences. 196, 01003 (2018) 
9. I.M. Zotov, A.P. Lapina, A.S. Chepurnenko, B.M. Yazyev, Materials Science Forum. 974, 551-555 (2019)

10. I.M. Zotov, A.P. Lapina, A.S. Chepurnenko, B.M. Yazyev, IOP Conference Series: Materials Science and Engineering. 661 (2019)

11. D. Gura, M. Kuzyakina, I. Gribkova, IOP Conference Series: Earth and Environmental Science 403(1), 012176 (2019). DOI: 10.1088/1755-1315/403/1/012176 